\title{
Differential Effects of Myopathy-Associated Caveolin-3 Mutants on Growth Factor Signaling
}

\author{
Eva Brauers, ${ }^{*}$ Agnes Dreier, ${ }^{*}$ Andreas Roos, ${ }^{\dagger}$ \\ Berthold Wormland, ${ }^{\ddagger}$ Joachim Weis, ${ }^{*}$ \\ and Alexander Krüttgen ${ }^{\S}$ \\ From the Institutes of Neuropathology, ${ }^{*}$ Human Genetics, ${ }^{\dagger}$ and \\ Medical Microbiology, Medical Faculty, Rheinisch-Westfälische \\ Technische Hochschule (RWTH) Aachen University, Aachen; and \\ the Department of Neurology, Klinikum Duisburg, \\ Duisburg, Germany
}

Caveolin-3 is an important scaffold protein of cholesterol-rich caveolae. Mutations of caveolin-3 cause hereditary myopathies that comprise remarkably different pathologies. Growth factor signaling plays an important role in muscle physiology; it is influenced by caveolins and cholesterol-rich rafts and might thus be affected by caveolin-3 dysfunction. Prompted by the observation of a marked chronic peripheral neuropathy in a patient suffering from rippling muscle disease due to the $R 26 Q$ caveolin-3 mutation and because TrkA is expressed by neuronal cells and skeletal muscle fibers, we performed a detailed comparative study on the effect of pathogenic caveolin-3 mutants on the signaling and trafficking of the TrkA nerve growth factor receptor and, for comparison, of the epidermal growth factor receptor. We found that the R26Q mutant slightly and the P28L strongly reduced nerve growth factor signaling in TrkA-transfected cells. Surface biotinylation experiments revealed that the R26Q caveolin-3 mutation markedly reduced the internalization of TrkA, whereas the P28L did not. Moreover, P28L expression led to increased, whereas R26Q expression decreased, epidermal growth factor signaling. Taken together, we found differential effects of the R26Q and P28L caveolin-3 mutants on growth factor signaling. Our findings are of clinical interest because they might help explain the remarkable differences in the degree of muscle lesions caused by caveolin-3 mutations and also the co-occurrence of peripheral neuropathy in the $R 26 Q$ caveolinopathy case presented. (Am J Pathol 2010, 177:261-270; DOI: 10.2353/ajpath.2010.090741)
Caveolae are flask-shaped invaginations of the plasma membrane implicated in membrane-trafficking and signal transduction. Caveolins are major scaffolding proteins of caveolae and lipid rafts. In these structures, cholesterol and lipid-modified signaling molecules such as Src-like kinases, H-Ras, and G-proteins accumulate. ${ }^{1}$ Caveolin-3 (M-Caveolin) is one of three members of the caveolin protein family. ${ }^{2}$ In contrast to caveolin-1 and caveolin-2, which are ubiquitously expressed, caveolin-3 is predominantly expressed in muscle fibers. The caveolin-3 proteins form homomeric complexes that are transported to the plasma membrane where they are associated with the dystrophin glycoprotein complex. ${ }^{3}$ Point mutations in caveolin-3 lead to a dissociation of the assembly process $^{4}$ and a decrease of sarcolemmal caveolin-3 levels. The caveolin-3 mutant R26Q has been shown to accumulate in the Golgi complex, ${ }^{5}$ which decreases the halflife of both the mutated and the wild-type-caveolin-3 protein. Interestingly, the different caveolin-3 mutations lead to a wide spectrum of phenotypes ranging from clinically asymptomatic hyperCKemia to rippling muscle disease, distal myopathy, and limb girdle muscular dystrophy type 1C. For instance, the point mutation R26Q can cause heterogeneous disease patterns encompassing this whole spectrum of disorders. ${ }^{3}$ In contrast, the mutation P28L, which is located in close proximity to mutation $R 26 Q$, causes persistent elevated serum levels of creatine kinase only, without overt clinical symptoms. ${ }^{6}$ The caveolin-3 mutation A45T leads to the same heterogeneous phenotype as R26Q except for distal myopathy, whereas the G55S mutation is associated with the limb girdle muscular dystrophy type $1 \mathrm{C}$ phenotype. ${ }^{7}$

Supported by the German Federal Ministry of Education and Research, Bundesministerium für Forschung und Technik (MD-Net Teilprojekt 22 01GM0601)

J.W. and A.K. contributed equally to this work.

Accepted for publication March 15, 2010.

Supplemental material for this article can be found on http://ajp. amjpathol.org.

Address reprint requests to Dr. Alexander Krüttgen, Institute of Medical Microbiology, Medical Faculty, Rheinisch-Westfälische Technische Hochschule (RWTH) Aachen University, Pauwelsstrasse 30, 52074 Aachen, Germany. E-mail: akruettgen@ukaachen.de. 
Growth factors that signal through cell surface receptors are concentrated in caveolae and lipid rafts. ${ }^{8}$ Therefore, mutations in caveolin-3 might lead to alterations in signaling and trafficking of these factors and their receptors. In line with this hypothesis, Parton and co-workers ${ }^{9}$ showed in $\mathrm{BHK}$ fibroblasts and PC12 cells that the C71W caveolin-3 mutant strongly diminished signaling of $\mathrm{H}$ Ras, an important downstream signal transducer of receptor tyrosine kinases. However, the consequences of caveolin-3 mutations on growth factor receptor activation at the cell surface are so far largely unexplored. Moreover, it is unclear how the different known caveolin-3 mutations trigger markedly different clinical phenotypes even when comparing cases with very similar mutations. This was recently exemplified by Minetti and co-workers, ${ }^{10}$ who showed that caveolin-3 T78M and T78K mutations lead to different phenotypes.

In the present study we describe muscle and nerve biopsy findings of a patient who suffered from both rippling muscle disease due to the R26Q caveolin-3 mutation and chronic peripheral neuropathy. We found differential effects of the R26Q and the P28L caveolin-3 point mutants on nerve growth factor (NGF) versus epidermal growth factor (EGF) signaling in our in vitro experiments. Our results give rise to the intriguing hypothesis that differential effects of caveolin-3 mutants on various growth factor signaling pathways are causally related to the so far unexplained differences in clinical phenotypes.

\section{Materials and Methods}

\section{Histology}

Paraffin and epoxy resin embedding and subsequent histological workup of the sural nerve and muscle biopsies as well as muscle biopsy immunohistochemistry was performed as described previously. ${ }^{11}$

\section{Cell Culture, Plasmids, and Transfection}

HEK293 cells (human embryonic kidney cells; provided by Dr. E. Shooter, Department of Neurobiology, Stanford University School of Medicine), $\mathrm{C}_{2} \mathrm{C}_{12}$ (mouse myoblasts; provided by American Type Culture Collection, Manassas, VA), PC12 cells (rat pheochromocytoma cells; provided by Dr. E. Shooter, Department of Neurobiology, Stanford University School of Medicine), and NIH3T3 cells (embryonic fibroblasts; provided by Dr. E. Dahl, Institute of Pathology, Medical Faculty RWTH Aachen University, Germany) were cultured in Dulbecco's modified Eagle's medium with 10\% fetal calf serum. Serum starvation of PC12 cells was performed by pre-incubation of cells with Dulbecco's modified Eagle's medium containing $0.5 \%$ bovine serum albumin (BSA; Roche, Mannheim, Germany) for 3 hours, whereas HEK293 cells were starved overnight in Dulbecco's modified Eagle's medium containing $0.5 \% \mathrm{BSA}$ to synchronize the cells to the GO/G1 phase of the cell cycle and to reduce background tyrosine phosphorylation caused by serum-containing medium. Cells were transfected by using Lipofectamine 2000 (Invitrogen, Carlsbad, CA) according to the manufacturer's protocol. The EGF receptor (EGFR) plasmid was kindly provided by Dr. M. E. Hegi (Université de Lausanne, Switzerland). Subcloning of EGFR cDNA into the pEXPR-IBA5 vector (IBA, Göttingen, Germany) was performed by using the restriction enzymes Xbal and Hindlll. The wild-typeCaveolin-3-HA construct was kindly provided by Drs. S. Roy and R. Parton (University of Queensland Medical School, Australia). To generate a green-fluorescent caveolin-3 plasmid, the wild-type-caveolin-3 cDNA was inserted in frame into pEGFP-N1 (Clontech Laboratories, Mountain View, CA) by using the restriction sites HindllI and BamHI. Constructs carrying the caveolin-3 point mutations R26Q, P28L, A45T, or G55S were created by using the QuikChange II SiteDirected Mutagenesis Kit (Stratagene, Cedar Creek, TX). The primer sequences to create the mutated constructs were as follows: R26Q-F: 5'-GATAGACTTGGTGAACCAAGACCCCAAGAACATCAATGAG-3'; R26Q-R: 5'-CTCATTGATGTTCTTGGGGTCTTGGTTCACCAAGTCTATC-3'; P28L-F: 5'-GACTTGGTGAACCGCGACCTCAAGAACATCAATGAGGACATTG-3'; P28L-R: 5'-CAATGTCCTCATTGATGTTCTTGAGGTCGCGGTTCACCAAGTC-3'; A45T-F: 5'-GATTTTGAAGACGTGATTACAGAGCCCGAGGGCACCTACAG-3'; A45T-R: 5'-CTGTAGGTGCCCTCGGGCTCTGTAATCACGTCTTCAAAATC-3'; G55S-F: 5'-GGCACCTACAGCTTCGACAGCGTATGGAAGGTGAGCTTCACCACG-3'; and G55S-R: 5'-CGTGGTGAAGCTCACCTTCCATACGCTGTCGAAGCTGTAGGTGCC-3'.

\section{Immunoblotting}

To prepare protein lysates of biopsies, thin cryostat sections of frozen skeletal muscle were sonicated and boiled for 5 minutes. After centrifugation, the supernatant was further processed. Transfected cells were placed on ice and washed once with cold PBS. Then, cold radioimmunoprecipitation assay lysis buffer (for all blots except caveolin-3 blots) containing protease inhibitor cocktail (Roche, Mannheim, Germany) and sodium-ortho-vanadate was added for 1 hour to lyse the cells on ice. For caveolin-3 immunoblots, T-PER (Pierce, Rockford, IL) was used as lysis buffer. After transferring cell lysates into precooled vials and centrifugation of the samples, the cell debris was discarded and the supernatant was transferred to new vials. Protein concentrations of lysates were quantified by using a Micro BCA protein assay (Pierce) according to the manufacturer's protocol. Adjusted amounts of lysates were denatured by boiling for 5 minutes in Laemmli buffer. Then, samples were subjected to SDS-polyacrylamide gel electrophoresis (PAGE) under reducing conditions and transferred to polyvinylidene difluoride -membranes (Millipore, Billerica, MA). Membranes were briefly stained with Ponceau $S$ to confirm transfer (Sigma-Aldrich, St. Louis, MO). Subsequently, the membranes for caveolin-3 immunoblots were blocked with $1 \%$ blocking buffer (Blocking reagent; Roche) overnight at $4^{\circ} \mathrm{C}$, whereas membranes for all other immunoblots were blocked in $5 \%$ low-fat dried milk in Tris-buffered saline with Tween (TBS-T) for 5 hours at $4^{\circ} \mathrm{C}$. After washing with TBS-T, the blots were incubated with the appropriate antibody for 2 hours or overnight at $4^{\circ} \mathrm{C}$, re- 
spectively. All primary antibodies were used at a 1:1000 dilution in TBS-T containing 3\% BSA. The following antibodies were used: rabbit anti-AKT IgG (Cell Signaling Technology, Danvers, MA); rabbit anti-pAKT (Cell Signaling Technology); rabbit anti-caveolin-3 (Abcam, Cambridge, UK); mouse anti-caveolin-3 (BD Bioscience Europe, Erembodegem, Belgium); rabbit anti-EGFR (Santa Cruz Biotechnology, Santa Cruz, CA); rabbit anti-phosphorylated EGFR (pEGFR; Cell Signaling Technology); rabbit anti-ERK (Cell Signaling Technology); mouse anti-pERK (Cell Signaling Technology); rabbit anti-TrkA (Upstate, Charlottesville, VA); and rabbit anti-pTrkA (Cell Signaling Technology). After washing with TBS-T, the membranes were incubated for 1 hour at room temperature with the secondary antibody conjugated to horseradish peroxidase (Pierce). Bound antibodies were detected by using a chemiluminescent substrate (Pierce). All immunoblotting experiments were performed three times with similar results.

\section{Surface Biotinylation Assay}

NGF rapidly triggers the internalization of its receptor TrkA and thus down-regulates TrkA levels on the surface of PC12 cells. Surface biotinylation of PC12 cells after stimulation with -/+NGF was performed as previously described. ${ }^{12}$ Briefly, PC12 cells were washed with cold PBS and then incubated with $250 \mu \mathrm{g} / \mathrm{ml}$ of the nonmembrane permeable crosslinker SULFO-NHS-biotin (Pierce) for 30 minutes on ice. Then, the cells were incubated with $10 \mathrm{mmol} / \mathrm{L}$ glycine (Sigma-Aldrich) in PBS for 20 minutes to quench the crosslinking reaction. Thereafter, the cells were washed with PBS; then the cells were lysed and biotinylated surface proteins were precipitated with streptavidin beads (Pierce) overnight, followed by SDSPAGE and immunoblotting against TrkA as described above. In a similar way, the assay was performed with EGFR-transfected HEK293 cells after stimulation with EGF. Surface biotinylation assays were performed three times with similar results.

\section{Immunofluorescence Staining}

$\mathrm{C}_{2} \mathrm{C}_{12}$ cells or NIH3T3 cells were grown on glass coverslips coated with Poly-L-Lysine (Sigma-Aldrich). After washing the cells one time with PBS, cells were fixed with $4 \%$ paraformaldehyde for 10 minutes. After 30 minutes of permeabilization with $0.1 \%$ Triton-X 100 and blocking with $10 \%$ goat serum (Sigma-Aldrich) diluted in PBS with $1 \%$ BSA, cells were rinsed with PBS and incubated with the primary antibody overnight at $4^{\circ} \mathrm{C}$ : rabbit anti-caveolin-3 (Abcam) and mouse anti-gm130 (BD Transduction Laboratories, Franklin Lakes, NJ) were diluted 1:500, whereas mouse anti-HA.11 (Covance, Berkeley, CA) was diluted 1:200 in 1\% BSA in PBS. After three washes with PBS, cells were incubated with the appropriate secondary antibody (labeled with AlexaFluor488 or AlexaFluor555; Molecular Probes, Carlsbad, CA) for 30 minutes at room temperature. After immunolabeling and washing for three times with PBS, the cells were mounted with Immu-mount (Thermo, Pittsburgh, PA) and examined by using an Axio- vert Apotome fluorescence microscope (Zeiss, Jena, Germany). All immunostaining experiments were performed at least 3 times with similar results.

\section{Results}

\section{Case Reports}

A 49-year-old patient presented with a history of chronically progressive distal sensory deficits associated with afferent tremor as well as paresis of the distal leg muscles. Symptoms had started to develop at age 25. There was no family history of neuromuscular disease. Biopsy of the left gastrocnemic muscle at the age of 39 had revealed chronic neurogenic atrophy combined with moderate necrotizing myopathy. The latter was initially thought to be secondary to the denervation atrophy. Concomitant biopsy of the left sural nerve demonstrated considerable loss of myelinated nerve fibers as well as groups of regenerating nerve fibers and axons with disproportionately thin myelin sheaths. These findings were consistent with a chronic, slowly progressive neuropathy predominantly of the axonal type combined accompanied by moderate demyelination. Minor cuffs of lymphocytes were present in the walls and in the immediate vicinity of epi- and endoneurial blood vessels. Inflammatory neuropathy was suspected, but repeated courses of therapeutic steroids only yielded temporary improvements. Clinical examination at age 49 revealed the above mentioned symptoms as well as generalized hypertrophy of the limb muscles. Percussion induced prominent abnormal muscle contractions ("rippling"). Plasma creatine kinase was elevated (567 U/L). Histological examination of biopsies of the gastrocnemic muscle and of the sural nerve of the contralateral leg at that time detected essentially the same features as detected 10 years earlier (Figure 1, A and B). Inflammatory infiltrates, however, were absent. Immunohistochemical analysis demonstrated absence of caveolin-3 immunoreactivity of normal sized and hypertrophic fibers, whereas atrophic fibers showed prominent caveolin-3 immunoreactivity (Figure 1, C and D). Dysferlin immunoreactivity was similar to the caveolin-3 pattern. Dystrophin (Dys 1-3) immunoreactivity of muscle fibers was moderately reduced; $\alpha-, \beta-, \gamma^{-}$, and $\delta$-sarcoglycan immunostaining was normal. Immunoblot analysis demonstrated no detectable caveolin-3 protein levels (Figure 1E). Sequencing of the caveolin-3 gene in DNA extracted from the unfixed muscle tissue revealed a heterozygous CGA to CAA base substitution in codon 26 causing the R26Q mutation. Microsatellite analysis and sequencing of the PMP22 gene and sequencing of the connexin-32, MPZ, and mitofusin-2 genes gave normal results.

\section{Different Apparent Molecular Weights of the R26Q and P28L Caveolin-3 Mutants in Transfected Cells}

The mutations R26Q, P28L, and A45T are located in the $\mathrm{N}$-terminal domain, whereas A45T is located within the 


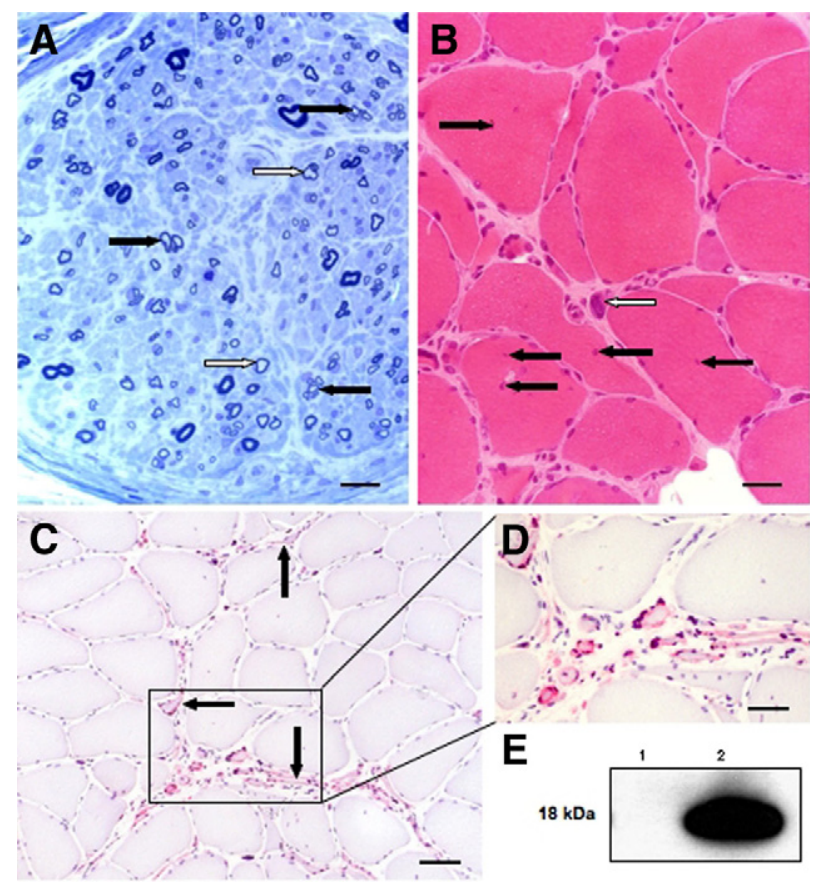

Figure 1. Sural nerve and gastrocnemius muscle biopsy features are shown. A: Sural nerve: Severe reduction in large myelinated nerve fiber density. Several small groups of regenerating axons (black arrows) and few large axons with disproportionately thin myelin sheaths (white arrows). Semithin section, toluidine blue. Scale bar $=30 \mu \mathrm{m}$. B: Marked muscle fiber atrophy with numerous flat/angular or rounded atrophic fibers, clumps of pycnotic nuclei in completely atrophic fibers (white arrow), and muscle fiber hypertrophy. Black arrows: nonsubsarcolemmal nuclei. In histochemical ATPase preparations, prominent fiber type grouping was present (not shown). Cryostat section, hematoxilin-eosin stain. Scale bar $=60 \mu \mathrm{m}$. C: Caveolin-3 immunoreactivity is absent in muscle fibers of normal size and in hypertrophic fibers. However, there is peculiar, strong caveolin-3 immunostaining (red) of the sarcolemma and/or the sarcoplasm of atrophic fibers (black arrows). Cryostat section, hematoxilin counterstain. Scale bar $=50 \mu \mathrm{m}$. D: Higher magnification of the region indicated in $\mathbf{C}$, demonstrating that the labeled structures are, in fact, atrophic skeletal muscle fibers. Scale bar $=30$ $\mu \mathrm{m}$. E: Immunoblot demonstrating absent caveolin-3 immunoreactivity in lane 1 loaded with patient muscle lysate. Lane 2 is loaded with normal human control muscle lysate.

signature sequence, a stretch of eight amino acids (FEDVIAEP) highly conserved in all three isoforms of caveolin (Figure 2). The G55S-amino acid change resides within the scaffolding region of the protein, which is part of the $\mathrm{N}$-terminal domain. To determine the effects of these pathogenic point mutations located in different functional domains of caveolin-3, we made use of transiently transfected HEK293 cells, a model cell line frequently used for the study of caveolin function. ${ }^{13-15}$ To compare the expression levels of wild-type-caveolin-3 and mutations

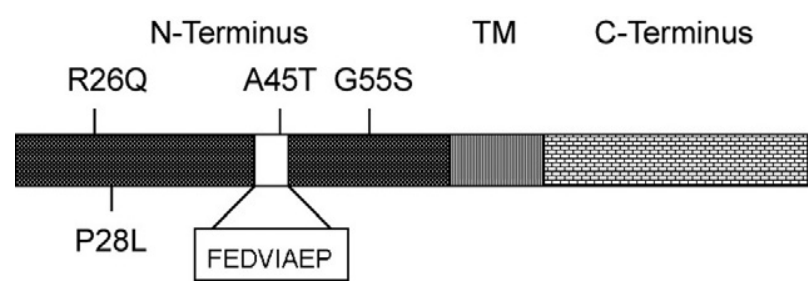

Figure 2. Schematic view of caveolin-3 domains and mutants. The point mutations R26Q, P28L, and A45T are localized in the N-terminal domain of the protein, whereas the amino acid change G55S is located in the scaffolding region. The point mutation $\mathrm{A} 45 \mathrm{~T}$ lies in a stretch of eight amino acids (FEDVIAEP) highly conserved in all three isoforms of caveolin.

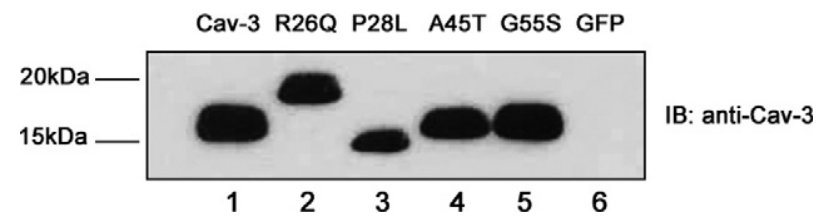

Figure 3. Expression of wild-type-caveolin-3 (wt-caveolin-3) and caveolin-3 mutants in HEK293 cells. HEK293 cells were transiently transfected with constructs encoding either the wild-type-caveolin-3 or the constructs carrying the mutations R26Q, P28L, A45T, G55S, or GFP as a mock-transfected negative control. The next day the cells were lysed and subjected to immunoblot analysis. Using a polyclonal antibody against caveolin-3, we evaluated the expression levels of the mutated proteins. The proteins carrying the mutations R26Q or P28L showed a surprising upward or downward mobility shift, respectively. IB, immunoblot.

thereof, HEK293 cells were either transiently transfected with wild-type-caveolin-3 or the constructs carrying the point mutations. As shown in Figure 3, immunoblot analysis of these cell lysates with antibodies directed against caveolin-3 revealed that the expression levels of R26Q, A45T, and G55S were similar to the wild-type protein expression levels. Interestingly, the protein carrying the mutation R26Q showed an upward shift, whereas the P28L mutant showed a downward shift in terms of their electrophoretic mobility, respectively, potentially due to mutation-induced conformational changes altering their migration on SDS-PAGE, as discussed below. Our observation of an altered mobility of R26Q confirms results of a previous study. ${ }^{5}$

\section{Different Subcellular Localizations of Mutated Caveolin-3 Variants}

By cotransfection of NIH3T3 cells with green fluorescent protein-tagged wild-type-caveolin-3 and each of the mutated caveolin-3 plasmids carrying an Hemagglutinin-tag as described above, we examined the influence of the caveolin-3 mutations on the subcellular localization of the wild-type-protein. ${ }^{5,16}$ As shown in Figure 4, the immunofluorescence staining of transfected NIH3T3 cells using a monoclonal antibody directed against the HA-tag attached to the mutated caveolin-3 proteins (shown in red, left and right columns, second-fifth row from the top) revealed that all mutated proteins showed a strong vesicular staining within transfected cells. Interestingly, wild-typecaveolin-3-GFP (shown in green, medium and right columns) showed a predominant surface localization only when cotransfected with wild-type-caveolin-3-HA (left column, first row). Moreover, cells expressing R26Q or G55S seemingly had higher surface levels of wild-type-cav-3-GFP than P28L and A45T.

To extend these results, we evaluated the subcellular distribution of the caveolin-3 mutants in a myoblast cell line. $\mathrm{C}_{2} \mathrm{C}_{12}$ cells were transiently transfected with wild-typecaveolin-3 or the mutated constructs and subjected to immunofluorescence analysis by using a rabbit antibody directed against caveolin-3 (green) and a mouse antibody against the Golgi marker gm130 (red). Nuclei were stained in blue (Hoechst 33342; Invitrogen). As shown in supplemental Figure 1 at http://ajp.amjpathol.org (A) wild-typecaveolin-3 is mostly localized to the plasma membrane 


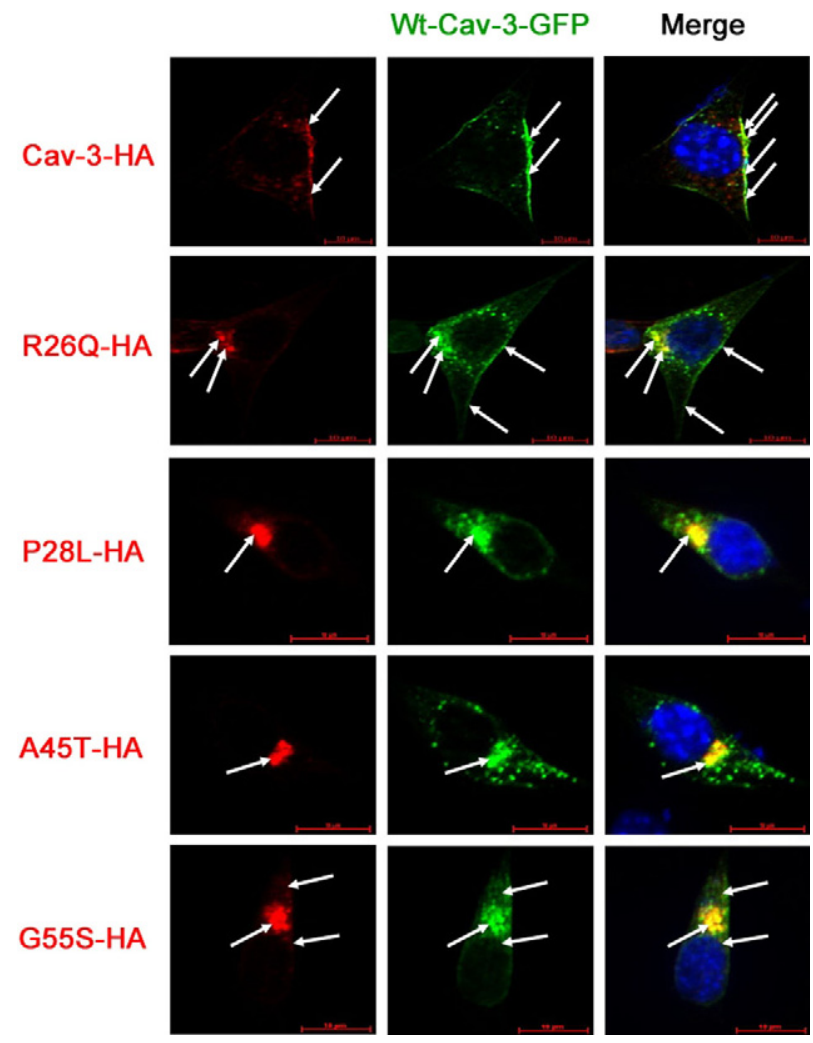

Figure 4. Analysis of NIH3T3 cells cotransfected with wild-type-caveolin-3 (wt-caveolin-3) and caveolin-3 mutants. NIH3T3 cells were transiently transfected either with both wild-type-caveolin-3 constructs carrying an HA- or GFP-tag or with wild-type-caveolin-3-GFP or mutated HA-constructs carrying the mutations R26Q, P28L, A45T, or G55S. One day after transfection, the cells were fixed with $4 \%$ paraformaldehyde and immunostained with a monoclonal mouse antibody against the HA-tag. In case of cotransfection of the wild-type-caveolin-3-GFP (green) and the constructs carrying the mutations P28L or A45T, less wild-type-caveolin-3 protein localizes to the plasma membrane than in cells co-expressing wild-type-caveolin-3-GFP and R26Q or G55S. White arrows indicate the localization of the wild-type-caveolin-3 or mutant cav-3 proteins. Scale bar $=10 \mathrm{um}$

(small arrows). In contrast, all mutated proteins showed prominent localization to the Golgi system (supplemental Figure 1 at http://ajp.amjpathol.org, B-E, large arrows) as indicated by the co-localization (yellow) of the proteins with the Golgi marker protein gm130. Interestingly, the predominant Golgi localization of R26Q, P28L, and A45T was associated with a diminished surface expression, whereas the G55S mutant also showed a wild-type-like localization to the cell surface (small arrows in A and E). This result indicates that different caveolin-3 mutations are differentially affected with respect to their vesicular transport in $\mathrm{C}_{2} \mathrm{C}_{12}$ cells.

\section{Differential Effects of the R26Q or P28L Caveolin-3 Mutants on Signal Transduction and Trafficking of TrkA}

To gain insight into the molecular pathology of the above described muscle diseases, we next investigated the potential influence of caveolin-3 on growth factor signaling. Based on encouraging results in initial experiments, we decided to focus on two of the four caveolin-3 mutants, the neighboring point mutants R26Q and P28L.

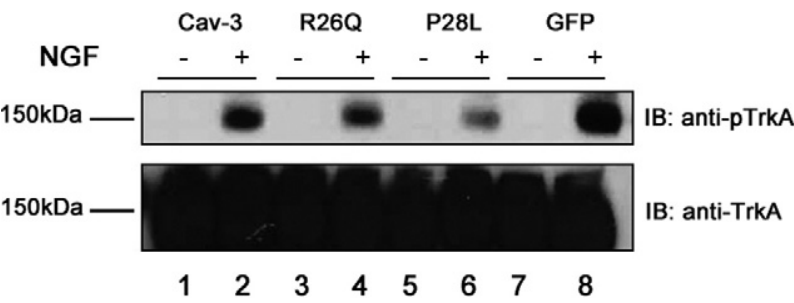

Figure 5. Analysis of NGF induced TrkA phosphorylation after transfection of cav-3 mutants in PC12 cells. PC12 cells were transiently transfected either with the wild-type-caveolin03 (wt-caveolin-3) construct or the constructs carrying the mutations R26Q, P28L, or GFP as a mock-transfected negative control. One day after transfection, the cells were prestarved for three hours and then stimulated with $50 \mathrm{ng} / \mathrm{ml}$ of NGF for five minutes. Subsequently, the cells were lysed, and lysates were subjected to Western blot analysis by using antibodies against TrkA (anti-TrkA) or antibodies against the Y490 phosphorylation site within the activation loop of TrkA (anti-pTrkA). After transfection of the caveolin-3 construct carrying the mutation P28L, TrkA phosphorylation levels are decreased compared with cells transfected with the wild-typeconstruct or the construct carrying the mutation R26Q. Loading controls were performed by probing with antibodies directed against TrkA. IB, immunoblot.

Caveolin-3 has been detected in central nervous system and peripheral nervous system tissue, in particular in astrocytes and Schwann cells. ${ }^{17-19}$ We found prominent nerve fiber alterations in nerve biopsies of a patient carrying the R26Q mutation (compare Figure 1). Previous studies revealed that TrkA is not exclusively expressed by neurons, but also by other cell types including reactive astrocytes ${ }^{20,21}$ as well as skeletal and smooth muscle fibers. $^{22,23}$ Therefore, we examined NGF-induced TrkA receptor tyrosine kinase signaling in the frequently used PC12 cell model system. ${ }^{24}$ PC12 cells harbor caveolae that contain TrkA, ${ }^{25}$ which is known to interact with caveolin-1. ${ }^{26}$ As caveolin-1 can form heterodimers with caveolin- $3,{ }^{27}$ we reasoned that transfection of $\mathrm{PC} 12$ cells with mutant caveolin-3 variants might affect NGF signaling.

Cells were transiently transfected with wild-typecaveolin-3 or mutants. Subsequently, we examined NGFinduced signaling by immunoblotting against phosphorylated downstream mediators. One day after transfection, cells were prestarved for 3 hours and then stimulated with NGF for 5 minutes. Immunoblotting by using antibodies against TrkA, tyrosine-phosphorylated TrkA (pTrkA), caveolin-3, and tubulin (caveolin-3, tubulin: data not shown) revealed that in cells transfected with P28L, TrkA phosphorylation was decreased compared with cells transfected with the wild-type-caveolin-3 construct (Figure 5, upper panel, compare lanes 2 and 6). On the other hand, transfection of the R26Q caveolin-3 construct had no significant effect on the phosphorylation of TrkA (Figure 5, compare lanes 2 and 4). Thus, given their close proximity, we were surprised to detect differential effects of these two caveolin-3 point mutations on TrkA phosphorylation (P28L $<\mathrm{R} 26 \mathrm{Q} \leq$ wild-type). To elucidate the effects of the caveolin-3 point mutations on the phosphorylation of kinases downstream of TrkA, we subsequently performed blots by using specific antibodies against phosphorylated Akt (also known as PKB) and ERK1/2 by using the same protein lysates as used in Figure 5. As shown in Figure 6, we observed strongly reduced levels of pAkt after transfection of the P28L mutant. In compar- 


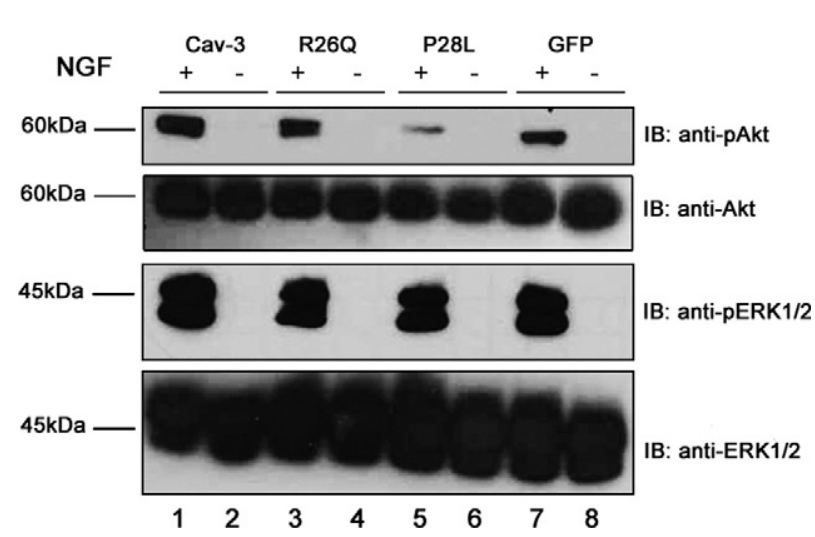

Figure 6. Analysis of the phosphorylation levels of Akt and ERK1/2. PC12 cells were transiently transfected either with the wild-type-caveolin-3 (wtcaveolin-3) construct or the constructs carrying the mutations R26Q, P28L, or GFP as a mock-transfected negative control. One day after transfection, the cells were prestarved for three hours and then stimulated with $50 \mathrm{ng} / \mathrm{ml}$ of NGF for five minutes. Subsequently the cells were lysed and subjected to Western blot analysis. Use of a polyclonal antibody against phosphorylated (pAkt) and unphosphorylated Akt (Akt) or phosphorylated ERK1/2 (pERK1/2) and ERK1/2 (ERK1/2) revealed lower phosphorylation levels of Akt after transfection of the construct carrying the mutation P28L than after transfection of the wild-type-construct or the construct carrying the $\mathrm{R} 26 \mathrm{Q}$ mutation. IB, immunoblot.

ison, transfection with $\mathrm{R} 26 \mathrm{Q}$ reduced Akt phosphorylation levels only to a minor extent. This result correlates with the pTrkA results presented earlier in Figure 5 (P28L $<\mathrm{R} 26 \mathrm{Q} \leq$ wild-type). However, it is interesting that we observed an effect of P28L solely on the levels of pAkt, but not on pERK1/2 (Figure 6, third panel from the top, compare lanes 1 and 5). These data suggest selective effects of certain caveolin-3 mutants on some, but not all downstream signaling pathways.

Trafficking of growth factor receptors is an emerging subject in the field of signal transduction. As trafficking of Trk receptors differentially regulates activation of ERK $1 / 2$ and $A k t,{ }^{28,29}$ we explored the effect of caveolin-3 on endocytosis of TrkA. It is known that after incubation of PC12 cells with NGF, the surface levels of TrkA are subjected to robust down-regulation due to endocytosis or these receptors into signaling endosomes. ${ }^{30}$ To detect potential effects of caveolin-3 mutations on TrkA endocytosis, we performed surface biotinylation assays as described previously. ${ }^{30,31}$ We transiently transfected PC12 cells with either the wild-type-caveolin-3 construct or the caveolin-3 construct carrying the point mutations R26Q or P28L. Twenty-four hours later, cells were prestarved for 3 hours and stimulated for 5 minutes with NGF. Subsequently the cells were surface-biotinylated, lysed, and the surface TrkA was pulled down with streptavidinbeads to selectively detect those TrkA receptors that had remained at the surface of the NGF stimulated cells. We found higher levels of TrkA at the plasma membrane of cells expressing R26Q than in cells expressing P28L (Figure 7, upper panel, compare lanes 3 and 5 for NGFstimulated cells and 4 and 6 for unstimulated cells). This indicates a differential effect of both mutants on TrkA surface trafficking and endocytosis. Interestingly, we also noticed that expression of wild-type-caveolin-3 increases the surface levels of TrkA compared with mock (GFP) transfected cells (compare lanes 2 and 8 for NGF-stimu-

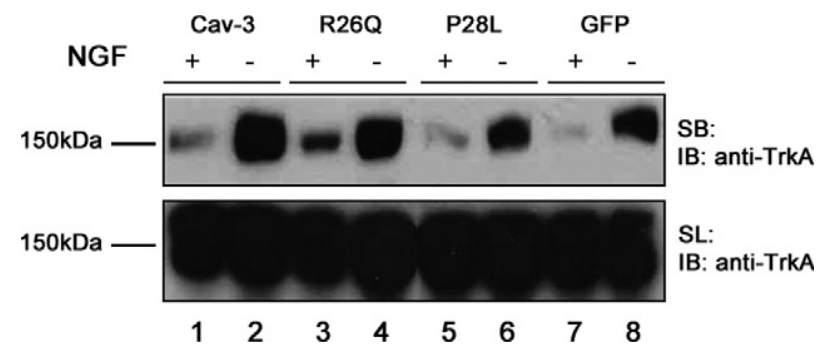

Figure 7. Surface biotinylation assay with PC12 cells transfected either with wild-type-caveolin-3 (wt-caveolin-3) or mutants. PC12 cells were transiently transfected either with the wild-type-caveolin-3 protein or the constructs carrying the mutation R26Q or P28L. Twenty-four hours later, the cells were prestarved for three hours and stimulated with $50 \mathrm{ng} / \mathrm{ml}$ of NGF. After stimulation, a surface biotinylation assay was performed to investigate if the caveolin-3 mutations influence the amount of surface TrkA levels. Western blot analysis of streptavidin precipitates revealed that higher levels of TrkA are present at the plasma membrane of stimulated PC12 cells expressing caveolin-3 R26Q than in cells expressing P28L. We also noticed that expression of wild-type-caveolin-3 increases the surface levels of TrkA compared with mock transfected cells (GFP). SB, surface biotinylation; SL, straight lysates; IB, immunoblot.

lated cells and 1 and 7 for unstimulated cells). On the other hand, the expression of wild-type-caveolin-3 and caveolin-3 mutants did not influence the surface levels of the Transferrin receptor, indicating specificity of the effects on TrkA surface trafficking/endocytosis (data not shown). Taken together, the surface TrkA levels after NGF addition showed clear differences between different caveolin-3 variants (P28L $<R 26 \mathrm{Q} \leq$ wild-type). Because this result correlates well with the results of the pTrkA blots (compare Figure 5), the altered internalization and altered signaling of TrkA in caveolin-3-expressing cells might be causally related.

\section{Influence of the R26Q or P28L Caveolin-3 Mutants on EGFR Signaling and Trafficking}

We next examined the effects of both caveolin-3 mutants on the signal transduction of another important receptor tyrosine kinase, EGFR. ${ }^{32}$ In a similar way as for TrkA (see above), we transiently cotransfected cells with the EGFR expression plasmid and the wild-type-caveolin-3 construct or those carrying the point mutations R26Q or P28L. After transfection, the cells were prestarved overnight to decrease background phosphorylation ${ }^{33}$ and then stimulated with EGF for 10 minutes, and the cells were lysed. Equal amounts of protein lysates were subjected to SDS-PAGE. Immunoblotting by using antibodies against tyrosine-pEGFR revealed that expression of $R 26 Q$ is associated with slightly lower levels of pEGFR in comparison with cells expressing wild-type-caveolin-3 (Figure 8, upper panel, compare lanes 1 and 3). Surprisingly, P28L expression strongly increased pEGFR in comparison with wild-type-caveolin-3 transfected cells (compare lanes 1 and 5) while even increasing pEGFR levels in comparison with mock (GFP)-transfected cells (compare lanes 5 and 7). Wild-type-caveolin-3 transfection apparently decreased the phosphorylation of EGFR that is possibly due to the overexpression of the protein. Interestingly, the expression of caveolin-3 variants altered the total levels of EGFR in cell lysates (Figure 8, 


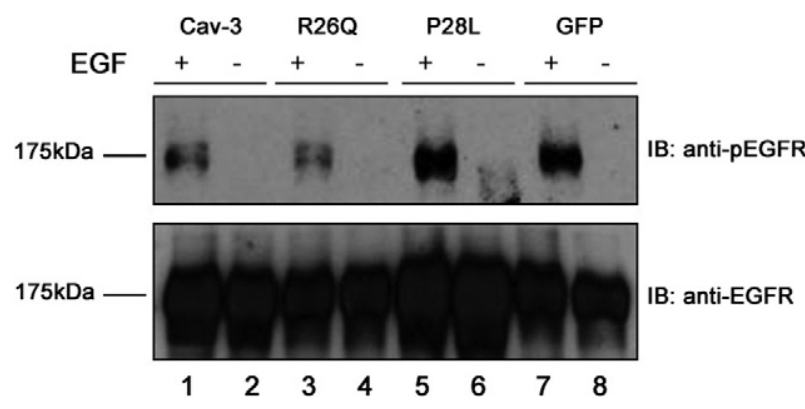

Figure 8. Analysis of the phosphorylation levels of EGFR after cotransfection of EGFR and caveolin-3 constructs in HEK293 cells. HEK293 cells were transiently cotransfected with the wild-type-caveolin-3 (wt-caveolin-3) construct and an EGFR construct, or EGFR and the constructs carrying the point mutations R26Q, P28L, or GFP as a negative control (GFP). Five hours after transfection, cells were prestarved overnight and then stimulated with 50 $\mathrm{ng} / \mathrm{ml} \mathrm{EGF}$ for ten minutes. Immunoblotting of same amounts of protein lysates by using antibodies against tyrosine-phosphorylated EGFR (pEGFR) revealed that transfection of $\mathrm{R} 26 \mathrm{Q}$ slightly decreased, whereas P28L clearly increased the levels of pEGFR. As a loading control, we probed with antiEGFR (lower panel).

lower panel), with P28L somewhat increasing the levels of EGFR. Taken together, we found differential effects of the two caveolin-3 mutations on the signaling of EGFR (R26Q $\leq$ wild-type $<$ P28L).

Next, we analyzed potential effects of caveolin mutations on EGFR endocytosis. In a similar way as described above for TrkA (compare Figure 7), we examined the surface levels of EGFR in HEK293 cells co-expressing EGFR and caveolin-3 constructs. After transfection, cells were prestarved overnight. The next day, they were stimulated with EGF for 10 minutes and a surface biotinylation assay was performed as described above. As shown in Figure 9, we found lower levels of EGFR at the surface of cells expressing R26Q and wild-type-caveolin-3 than in cells expressing the P28L or mock (GFP)-transfected control cells (R26Q $\leq$ wild-type $<$ P28L). This pattern correlates well with the differences in terms of pEGFR described above (compare Figure 8).

In a similar way as described for TrkA (see above), we subsequently examined the effects of both caveolin-3

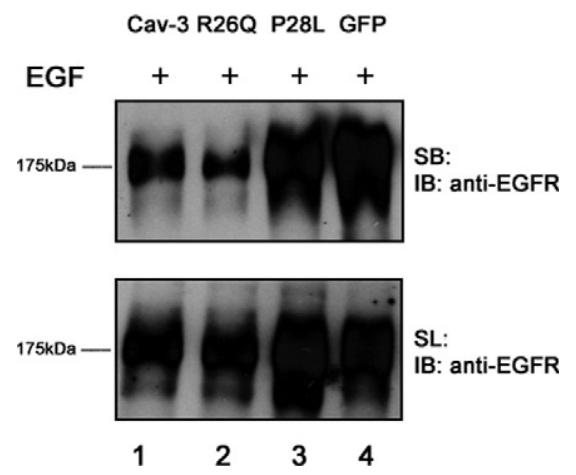

Figure 9. Analysis of the phosphorylation levels of ERK1/2 and Akt after cotransfection of EGFR and caveolin-3 constructs in HEK293 cells. HEK293 cells were transiently cotransfected with the wild-type-caveolin-3 construct and an EGFR construct, or EGFR and the constructs carrying the point mutations R26Q, P28L, or GFP as a negative control. Five hours after transfection, cells were prestarved overnight and then stimulated with $50 \mathrm{ng} / \mathrm{ml}$ EGF for ten minutes. Immunoblotting of same amounts of protein lysates by using antibodies against phosphorylated ERK1/2 (pERK1/2) and phosphorylated Akt (pAkt) revealed that transfection of both R26Q and P28L did not lead to major alterations. IB, immunoblot.

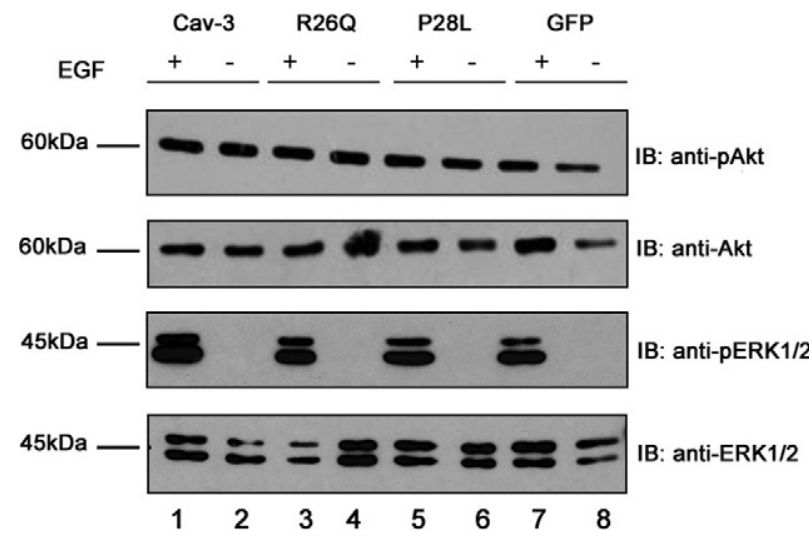

Figure 10. Surface biotinylation assay of HEK 293 cells cotransfected with EGFR and either wild-type-caveolin-3 (wt-caveolin-3) or the mutants. HEK293 cells were transiently transfected with the EGFR-construct and either with the wild-type-caveolin-3 protein or the constructs carrying the mutation R26Q or P28L (or GFP as a mock-transfected negative control). The transfected cells were prestarved overnight and stimulated with $100 \mathrm{ng} / \mathrm{ml} \mathrm{EGF}$ for ten minutes. After stimulation, a surface biotinylation assay was performed to investigate if the mutations in caveolin-3 influence the amount of surface EGFR levels. Western blot analysis revealed that slightly lower levels of EGFR are present at the plasma membrane of EGF-stimulated HEK293 cells expressing caveolin-3 R26Q than in cells expressing the wild-type-construct. $\mathrm{SB}$, surface biotinylation; SL, straight lysate; IB, immunoblot.

mutants on ERK $1 / 2$ and Akt downstream of EGFR. We detected no apparent differences regarding the phosphorylation levels of both ERK1/2 and Akt (Figure 10). This contrasts with our earlier results of altered TrkA downstream signaling in cells expressing the protein carrying the P28L mutation (compare Figure 6).

In summary, compared with wild-type-caveolin-3, R26Q slightly impairs EGFR signaling, whereas P28L strongly increases EGFR signaling. In contrast, R26Q slightly decreases TrkA signaling, whereas P28L strongly decreases TrkA signaling ( $p E G F R$ : R26Q $\leq$ wild-type < P28L and pTrkA: P28L $<$ R26Q $\leq$ wild-type). Interestingly, overexpression of wild-type-caveolin-3 decreased both pTrkA and pEGFR levels.

\section{Discussion}

Mutations in caveolin-3 cause an array of diverse, clinically asymptomatic to severe myopathies. Understanding the pathomechanisms of caveolinopathies requires detailed information on the functions of caveolin-3. Many different potential functions for caveolae and caveolins have been suggested, ${ }^{34}$ including the hypothesis that caveolins serve as signaling scaffolds for growth factor receptors. ${ }^{35}$ In line with this, many authors reported on the dependence of signaling receptors on cholesterolrich rafts, and one article revealed the effects of $\mathrm{C} 71 \mathrm{~W}$ mutated caveolin-3 on important signal transduction pathways. ${ }^{9}$ However, there is a divided opinion on the role of caveolae and lipid-rafts in receptor tyrosine kinase signaling, with some authors suggesting that rafts/caveolae inhibit RTK signaling, whereas others support the opposite view. ${ }^{36,37}$ In the present study, wild-type-caveolin-3 transfection decreased TrkA signaling and EGFR signaling in comparison with mock transfected cells. Thus, our results are in agreement with those previous 
studies that revealed an inhibitory effect of wild-typecaveolin on TrkA signaling ${ }^{26}$ and EGFR signaling. ${ }^{38-40}$

However, prompted by the observation of a prominent peripheral neuropathy in a R26Q caveolinopathy patient, the main focus of the present study was to perform a comparative survey of the effects of two myopathy-associated caveolin-3 mutants (P28L and R26Q) on the signal transduction of two important prototypical RTKs, TrkA and EGFR. We found several interesting dichotomies concerning the signaling and trafficking of these both RTKs after transfection of the caveolin-3 mutations. Although P28L strongly decreased NGF-induced phosphorylation of TrkA and the phosphorylation of the downstream kinase Akt (without affecting the phosphorylation of ERK 1/2), the neighboring R26Q mutation had only slight effects on TrkA phosphorylation. Moreover, R26Q expression slightly reduced the EGF-induced phosphorylation of the EGFR, whereas P28L strongly enhanced EGF-induced phosphorylation of EGFR (pEGFR: R26Q $\leq$ wild-type < P28L and pTrkA: P28L < R26Q $\leq$ wild-type). On the other hand, TrkA internalization was severely impaired by R26Q, but not by P28L caveolin-3, suggesting that the R26Q mutation might affect neurotrophin receptor trafficking and thus providing a potential explanation for the marked chronic peripheral neuropathy observed in our patient. In line with this hypothesis, an impaired activity of the caveolin-3 homolog caveolin-1 has recently been implicated in alterations of neurotrophic mechanisms in Schwann cells leading to polyneuropathy. ${ }^{41}$

In conclusion, our novel finding of various effects of caveolin-3 mutants on different RTKs might contribute to understanding the puzzling observation that neighboring caveolin-3 point mutations cause diverse phenotypes in patients. Moreover, individual genetic differences caused by single nucleotide polymorphisms in genes for growth factor receptors or cholesterol-synthesis associated genes could play an additional role in the pathogenesis of caveolinopathies, ${ }^{42}$ contributing to the puzzling differences between clinical phenotypes. ${ }^{43}$

\section{Caveolin-3 Mutations Lead to Alterations in Their Apparent Molecular Weight}

An upward shift of the caveolin-3 R26Q mutant in SDSPAGE has been described earlier. ${ }^{5}$ However, one remarkable finding that emerged from our immunoblots is the difference in apparent molecular weight of R26Q and P28L in SDS-PAGE. A significantly altered running behavior triggered by single point mutations in other proteins (such as ciliary neurotrophic factor) ${ }^{44}$ has sometimes been reported in the literature. The reason could be an altered structure of mutated proteins that is not completely unfolded by boiling in Laemmli buffer containing dithiothreitol. Another possibility to explain the increased apparent molecular weight could be posttranslational modifications such as phosphorylation, acetylation, ubiquitination, lipid modifications (leading to increased apparent molecular weight), or proteolytical cleavage (implicating a decrease in apparent molecular weight). Therefore, we used prediction programs available at the
Swiss-Prot/ExPASy webpage to explore the possibility that the two caveolin-3 point mutations might cause different alterations of conformation and posttranslational modification, respectively. This preliminary analysis did not reveal any obvious differences that might explain our observation. Further studies (such as mass spectrometric analysis of the R26Q and P28L bands) should shed light on this issue.

\section{Caveolin-3 Mutations Influence the Localization of Wild-Type-Caveolin-3}

Our cotransfection experiments with wild-type-caveolin-3 and the mutated constructs revealed that the mutations P28L and A45T act in a dominant negative way as already described for the mutation P104L. ${ }^{16}$ P28L and A45T lead to diminished surface location of wild-typecaveolin-3, whereas the mutations R26Q and G55S seem to have a much less dramatic effect on the wild-typecaveolin-3 surface levels, although also these mutants trigger an accumulation of wild-type-caveolin-3 within cells. ${ }^{5}$ Therefore, we hypothesize that various amounts of wild-type-caveolin-3 at different subcellular localizations (plasma membrane versus Golgi) might trigger different phenotypes of the disease. How might this mislocalization of wild-type-caveolin-3 cause altered RTK signaling? Interestingly, it is known that RTK activation (such as TrkA ${ }^{45}$ ) does not only occur at the plasma membrane but also in the Golgi apparatus, influencing key signaling mediators like Akt and ERK1/2 at both subcellular localizations, as discussed below. Thus, altered wild-typeprotein levels triggered by different caveolin- 3 mutants at the plasma membrane or the Golgi might differentially affect the signaling of different RTKs. However, additional disease modifiers unrelated to caveolin-3 might as well contribute to the diversity of the clinical phenotypes. ${ }^{46}$

\section{Differential in Vitro Effects of Different Caveolin-3 Mutants on Growth Factor Signaling}

What might be the mechanism of caveolin-3 mutation induced effects on RTK signaling? We found an intriguing concordance of results from independent assays assessing the trafficking and signal transduction of two RTKs (EGFR: R26Q $\leq$ wild-type < P28L and TrkA: P28L < $\mathrm{R} 26 \mathrm{Q} \leq$ wild-type), indicating that the caveolin-3 effects on trafficking and signaling are mechanistically linked. Thus, we speculate that caveolin-3 mutants alter the trafficking of RTKs, thereby altering their signaling. The effects of caveolin- 3 on RTK trafficking and signaling might be direct (via altered protein: protein interaction of caveolin, RTK) or indirect (such as via altered cholesterol trafficking, which in turn is known to affect RTK signaling). However, our finding that caveolin-3 mutants decrease the surface levels of these RTKs provides an additional plausible explanation for the lower responsiveness of RTKs in caveolin-3 mutant cells. Intriguingly, all caveolin-3 mutants examined by us showed strong localization to the Golgi apparatus and, with exception of the G55S 
mutant, only minor localization to the plasma membrane. Thus, we speculate that a traffic jam within the secretory apparatus might explain the lower surface levels of RTKs, as these receptors need to pass through the Golgi system to reach the cell surface. ${ }^{16}$ However, it is surprising that both R26Q and P28L accumulate within the Golgi, whereas surface levels of EGFR were slightly reduced in cells transfected with R26Q while surface levels of EGFR were actually increased by P28L. Perhaps, these two caveolin-3 mutants exert their effects on EGFR via different mechanisms. The R26Q mutant might directly diminish the surface trafficking of EGFR via direct caveolin: EGFR retention in the Golgi apparatus, whereas the P28L mutant might exert indirect effects on EGFR signaling, such as altering cholesterol transport or glycosylation of EGFR, which is known to occur in the Golgi apparatus. ${ }^{47}$ Thereby P28L could indirectly alter EGFR signaling after successful surface trafficking of this RTK. Further detailed studies on receptor traffic and cholesterol synthesis in relation to caveolin-3 are necessary to address these issues. Because lipid rafts regulate the binding affinity of EGFR to EGF, another interesting possibility might be that different caveolin-3 mutants differentially affect the binding affinity of RTKs for their ligands, ${ }^{48}$ which leads then to aberrant signaling and trafficking of the receptor molecules.

\section{Differential Effects of the R26Q and P28L Mutations on EGFR and Trk Signaling: Correlation with Clinical Observations in Myopathy Patients}

The phenotype of patients carrying the R26Q mutation (according to our in vitro signaling results: slightly diminished TrkA signaling and diminished EGFR signaling) is more severe compared with the P28L patients described so far (according to our in vitro signaling results: diminished TrkA signaling and enhanced EGFR signaling). ${ }^{6,49}$ EGFR is expressed by myoblasts and might thus play a role during muscle development, differentiation, and regeneration. ${ }^{50}$ In the context of the rippling phenomenon caused by the R26Q caveolin-3 mutation, it is of interest that caveolin-3 immunoreactivity has been shown to accumulate at neuromuscular junctions and to vanish after denervation. ${ }^{51}$ Regarding neurotrophins, the R26Q mutation and its effects on TrkA internalization could be of pathophysiological significance because the Trk receptor family is important for the development and maintenance of the neuromuscular junction. ${ }^{52,53}$

\section{Acknowledgments}

We thank Mrs. Elke Beck and Mrs. Eveline Pascual for expert technical assistance, and Dr. Thomas Nachreiner (RWTH Aachen University, Germany) for support in figure layout. The wild-type caveolin-3 plasmid was kindly provided by Drs. Sandrine Roy and Robert G. Parton (University of Queensland Medical School, Australia), the EGFR plasmid by Dr. Monika E. Hegi (University of Lausanne,
Switzerland), and the TrkAll plasmid by Dr. Phil Barker (McGill University, Montreal, Canada).

\section{References}

1. Lisanti MP, Sargiacomo M, Scherer PE: Purification of caveolaederived membrane microdomains containing lipid-anchored signaling molecules, such as GPI-anchored proteins. H-Ras, Src-family tyrosine kinases, eNOS, and G-protein alpha-, beta-, and gammasubunits. Methods Mol Biol 1999, 116:51-60

2. Way M, Parton RG: M-caveolin, a muscle-specific caveolin-related protein. FEBS Lett 1996, 378:108-112

3. Vorgerd M, Ricker K, Ziemssen F, Kress W, Goebel HH, Nix WA, Kubisch C, Schoser BG, Mortier W: A sporadic case of rippling muscle disease caused by a de novo caveolin-3 mutation. Neurology 2001, 57:2273-2277

4. Herrmann R, Straub V, Blank M, Kutzick C, Franke N, Jacob EN Lenard HG, Kroger S, Voit T: Dissociation of the dystroglycan complex in caveolin-3-deficient limb girdle muscular dystrophy. Hum Mol Genet 2000, 9:2335-2340

5. Sotgia F, Woodman SE, Bonuccelli G, Capozza F, Minetti C, Scherer PE, Lisanti MP: Phenotypic behavior of caveolin-3 R26Q, a mutant associated with hyperCKemia, distal myopathy, and rippling muscle disease. Am J Physiol Cell Physiol 2003, 285:C1150-C1160

6. Merlini L, Carbone I, Capanni C, Sabatelli P, Tortorelli S, Sotgia F Lisanti MP, Bruno C, Minetti C: Familial isolated hyperCKaemia associated with a new mutation in the caveolin-3 (CAV-3) gene. J Neurol Neurosurg Psychiatry 2002, 73:65-67

7. McNally EM, de Sa Moreira E, Duggan DJ, Bonnemann CG, Lisanti MP, Lidov HG, Vainzof M, Passos-Bueno MR, Hoffman EP, Zatz M, Kunkel LM: Caveolin-3 in muscular dystrophy. Hum Mol Genet 1998, 7:871-877

8. Felberbaum-Corti M, Van Der Goot FG, Gruenberg J: Sliding doors: clathrin-coated pits or caveolae? Nat Cell Biol 2003, 5:382-384

9. Carozzi AJ, Roy S, Morrow IC, Pol A, Wyse B, Clyde-Smith J, Prior IA, Nixon SJ, Hancock JF, Parton RG: Inhibition of lipid raft-dependent signaling by a dystrophy-associated mutant of caveolin-3. J Biol Chem 2002, 277:17944-17949

10. Traverso M, Gazzerro E, Assereto S, Sotgia F, Biancheri R, Stringara S, Giberti L, Pedemonte M, Wang X, Scapolan S, Pasquini E, Donati MA, Zara F, Lisanti MP, Bruno C, Minetti C: Caveolin-3 T78M and T78K missense mutations lead to different phenotypes in vivo and in vitro. Lab Invest 2008, 88:275-283

11. Weis J, Kaussen M, Calvo S, Buonanno A: Denervation induces a rapid nuclear accumulation of MRF4 in mature myofibers. Dev Dyn 2000, 218:438-451

12. Saxena S, Howe CL, Cosgaya JM, Steiner P, Hirling H, Chan JR, Weis J, Kruttgen A: Differential endocytic sorting of p75NTR and TrkA in response to NGF: a role for late endosomes in TrkA trafficking. Mol Cell Neurosci 2005, 28:571-587

13. Bhatnagar A, Sheffler DJ, Kroeze WK, Compton-Toth B, Roth BL: Caveolin-1 interacts with 5-HT2A serotonin receptors and profoundly modulates the signaling of selected Galphaq-coupled protein receptors. J Biol Chem 2004, 279:34614-34623

14. Wharton J, Meshulam T, Vallega G, Pilch P: Dissociation of insulin receptor expression and signaling from caveolin-1 expression. J Biol Chem 2005, 280:13483-13486

15. Sotgia F, Bonuccelli G, Minetti C, Woodman SE, Capozza F, Kemp RG, Scherer PE, Lisanti MP: Phosphofructokinase muscle-specific isoform requires caveolin-3 expression for plasma membrane recruitment and caveolar targeting: implications for the pathogenesis of caveolin-related muscle diseases. Am J Pathol 2003, 163:2619-2634

16. Galbiati F, Volonte D, Minetti C, Chu JB, Lisanti MP: Phenotypic behavior of caveolin-3 mutations that cause autosomal dominant limb girdle muscular dystrophy (LGMD-1C): retention of LGMD-1C caveolin-3 mutants within the golgi complex. J Biol Chem 1999, 274:25632-25641

17. Shin T, Kim H, Jin JK, Moon C, Ahn M, Tanuma N, Matsumoto Y: Expression of caveolin-1, -2 , and -3 in the spinal cords of Lewis rats with experimental autoimmune encephalomyelitis. J Neuroimmunol 2005, 165:11-20

18. Nishiyama K, Trapp BD, Ikezu T, Ransohoff RM, Tomita T, Iwatsubo T, Kanazawa I, Hsiao KK, Lisanti MP, Okamoto T: Caveolin-3 upregulation 
activates beta-secretase-mediated cleavage of the amyloid precursor protein in Alzheimer's disease. J Neurosci 1999, 19:6538-6548

19. Ikezu T, Ueda H, Trapp BD, Nishiyama K, Sha JF, Volonte D, Galbiati F, Byrd AL, Bassell G, Serizawa H, Lane WS, Lisanti MP, Okamoto T: Affinity-purification and characterization of caveolins from the brain: differential expression of caveolin-1, -2 , and -3 in brain endothelial and astroglial cell types. Brain Res 1998, 804:177-192

20. Oderfeld-Nowak B, Orzylowska-Sliwinska O, Soltys Z, Zaremba M, Januszewski S, Janeczko K, Mossakowski M: Concomitant up-regulation of astroglial high and low affinity nerve growth factor receptors in the CA1 hippocampal area following global transient cerebral ischemia in rat. Neuroscience 2003, 120:31-40

21. Aguado F, Ballabriga J, Pozas E, Ferrer I: TrkA immunoreactivity in reactive astrocytes in human neurodegenerative diseases and colchicine-treated rats. Acta Neuropathol 1998, 96:495-501

22. Rende M, Brizi E, Conner J, Treves S, Censier K, Provenzano C, Taglialatela G, Sanna PP, Donato R: Nerve growth factor (NGF) influences differentiation and proliferation of myogenic cells in vitro via TrKA. Int J Dev Neurosci 2000, 18:869-885

23. Shibayama E, Koizumi H: Cellular localization of the Trk neurotrophin receptor family in human non-neuronal tissues. Am J Pathol 1996, 148:1807-1818

24. Segal RA, Greenberg ME: Intracellular signaling pathways activated by neurotrophic factors. Annu Rev Neurosci 1996, 19:463-489

25. Peiro S, Comella JX, Enrich C, Martin-Zanca D, Rocamora N: PC12 cells have caveolae that contain TrkA: caveolae-disrupting drugs inhibit nerve growth factor-induced, but not epidermal growth factor-induced, MAPK phosphorylation. J Biol Chem 2000, 275:37846-37852

26. Bilderback TR, Gazula VR, Lisanti MP, Dobrowsky RT: Caveolin interacts with Trk $A$ and $p 75(N T R)$ and regulates neurotrophin signaling pathways. J Biol Chem 1999, 274:257-263

27. Volonte D, McTiernan CF, Drab M, Kasper M, Galbiati F: Caveolin-1 and caveolin-3 form heterooligomeric complexes in atrial cardiac myocytes that are required for doxorubicin-induced apoptosis. Am J Physiol Heart Circ Physiol 2008, 294:H392-H401

28. Moises T, Dreier A, Flohr S, Esser M, Brauers E, Reiss K, Merken D, Weis J, Kruttgen A: Tracking TrkA's trafficking: nGF receptor trafficking controls NGF receptor signaling. Mol Neurobiol 2007, 35:151-159

29. Bronfman FC, Escudero CA, Weis J, Kruttgen A: Endosomal transport of neurotrophins: roles in signaling and neurodegenerative diseases. Dev Neurobiol 2007, 67:1183-1203

30. Saxena S, Bucci C, Weis J, Kruttgen A: The small GTPase Rab7 controls the endosomal trafficking and neuritogenic signaling of the nerve growth factor receptor TrkA. J Neurosci 2005, 25:10930-10940

31. Moises T, Wuller S, Saxena S, Senderek J, Weis J, Kruttgen A: Proteasomal inhibition alters the trafficking of the neurotrophin receptor TrkA. Biochem Biophys Res Commun 2009, 387:360-364

32. Ullrich A, Coussens L, Hayflick JS, Dull TJ, Gray A, Tam AW, Lee J, Yarden Y, Libermann TA, Schlessinger J, Downward J, Mayes ELV, Whittle N, Waterfield MD, Seeburg PH: Human epidermal growth factor receptor cDNA sequence and aberrant expression of the amplified gene in A431 epidermoid carcinoma cells. Nature 1984, 309:418-425

33. Kiel C, Serrano L: Cell type-specific importance of ras-c-raf complex association rate constants for MAPK signaling. Sci Signal 2009, 2:ra38

34. Simons K, Toomre D: Lipid rafts and signal transduction. Nat Rev Mol Cell Biol 2000, 1:31-39

35. Woodman SE, Sotgia F, Galbiati F, Minetti C, Lisanti MP: Caveolinopathies: mutations in caveolin-3 cause four distinct autosomal dominant muscle diseases. Neurology 2004, 62:538-543
36. Paratcha G, Ibanez CF: Lipid rafts and the control of neurotrophic factor signaling in the nervous system: variations on a theme. Curr Opin Neurobiol 2002, 12:542-549

37. Evangelopoulos ME, Weis J, Kruttgen A: Signalling pathways leading to neuroblastoma differentiation after serum withdrawal: $\mathrm{hDL}$ blocks neuroblastoma differentiation by inhibition of EGFR. Oncogene 2005, 24:3309-3318

38. Couet J, Sargiacomo M, Lisanti MP: Interaction of a receptor tyrosine kinase, EGF-R, with caveolins Caveolin binding negatively regulates tyrosine and serine/threonine kinase activities. J Biol Chem 1997, 272:30429-30438

39. Mineo C, Gill GN, Anderson RG: Regulated migration of epiderma growth factor receptor from caveolae. J Biol Chem 1999, 274:30636-30643

40. Wang XQ, Yan Q, Sun P, Liu JW, Go L, McDaniel SM, Paller AS: Suppression of epidermal growth factor receptor signaling by protein kinase C-alpha activation requires CD82, caveolin-1, and ganglioside. Cancer Res 2007, 67:9986-9995

41. McGuire JF, Rouen S, Siegfreid E, Wright DE, Dobrowsky RT: Caveolin-1 and altered neuregulin signaling contribute to the pathophysiological progression of diabetic peripheral neuropathy. Diabetes 2009, 58:2677-2686

42. Savas S, Geraci J, Jurisica I, Liu G: A comprehensive catalogue of functional genetic variations in the EGFR pathway: protein-protein interaction analysis reveals novel genes and polymorphisms important for cancer research. Int J Cancer 2009, 125:1257-1265

43. Dobrowsky RT, Rouen S, Yu C: Altered neurotrophism in diabetic neuropathy: spelunking the caves of peripheral nerve. J Pharmacol Exp Ther 2005, 313:485-491

44. Panayotatos N, Radziejewska E, Acheson A, Pearsall D, Thadani A, Wong V: Exchange of a single amino acid interconverts the specific activity and gel mobility of human and rat ciliary neurotrophic factors. J Biol Chem 1993, 268:19000-19003

45. Sorkin A: TRKing signals through the Golgi. Sci STKE 2005, 2005:pe1

46. de Paula F, Vainzof M, Bernardino AL, McNally E, Kunkel LM, Zatz M: Mutations in the caveolin-3 gene: when are they pathogenic? Am J Med Genet 2001, 99:303-307

47. Lajoie P, Partridge EA, Guay G, Goetz JG, Pawling J, Lagana A, Joshi B, Dennis JW, Nabi IR: Plasma membrane domain organization regulates EGFR signaling in tumor cells. J Cell Biol 2007, 179:341-356

48. den Hartigh JC, van Bergen en Henegouwen PM, Boonstra J, Verkleij AJ: Cholesterol and phosphoinositides increase affinity of the epidermal growth factor receptor. Biochim Biophys Acta 1993, 1148:249-256

49. Capanni C, Sabatelli P, Mattioli E, Ognibene A, Columbaro M, Lattanzi G, Merlini L, Minetti C, Maraldi NM, Squarzoni S: Dysferlin in a hyperCKaemic patient with caveolin 3 mutation and in $\mathrm{C} 2 \mathrm{C} 12$ cells after p38 MAP kinase inhibition. Exp Mol Med 2003, 35:538-544

50. Chen X, Raab G, Deutsch U, Zhang J, Ezzell RM, Klagsbrun M: Induction of heparin-binding EGF-like growth factor expression during myogenesis: activation of the gene by MyoD and localization of the transmembrane form of the protein on the myotube surface. J Biol Chem 1995, 270:18285-18294

51. Carlson BM, Carlson JA, Dedkov EI, McLennan IS: Concentration of caveolin-3 at the neuromuscular junction in young and old rat skeletal muscle fibers. J Histochem Cytochem 2003, 51:1113-1118

52. Ip FC, Cheung J, Ip NY: The expression profiles of neurotrophins and their receptors in rat and chicken tissues during development. Neurosci Lett 2001, 301:107-110

53. Pitts EV, Potluri S, Hess DM, Balice-Gordon RJ: Neurotrophin and Trk-mediated signaling in the neuromuscular system. Int Anesthesiol Clin 2006, 44(2):21-76 